\title{
FACTORES PREDICTORES DE SOLICITUD DE AYUDA PSICOLÓGICA EN LOS MESES INMEDIATOS AL DIAGNÓSTICO ONCOLÓGICO
}

\section{PREDICTIVE FACTORS FOR ASKING PSYCHOLOGICAL ASSISTANCE IN THE MONTHS FOLLOWING TO CANCER DIAGNOSIS}

\author{
Concha León 1 , Esther Jovell², Rosanna Mirapeix ${ }^{3}, A_{n}$ gels Arcusa ${ }^{4}$ y Tomás Blasco \\ 1 Institut Oncológic del Vallès [Consorci Sanitari de Terrasa-Corporació Sanitària Parc Tauli] \\ ${ }^{2}$ Consorci Sanitari de Terrassa \\ ${ }^{3}$ Hospital Parc Tauli de Sabadell \\ ${ }^{4}$ Consorci Sanitari de Terrassa. Institut Oncológic del Vallès \\ ${ }^{5}$ Departament de Psicologia Bàsica. Universitat Autònoma de Barcelona
}

\section{Resumen}

Objetivo: Determinar la prevalencia de pacientes que, tras conocer el diagnóstico oncológico y el tratamiento prescrito, desearían recibir atención psicooncológica, e identificar las características sociodemográficas, biomédicas y psicológicas de los mismos, con la finalidad de establecer un posible perfil del demandante.

Método: Se evaluaron 236 pacientes oncológicos atendidos en el Consorci Sanitari de Terrassa, mediante un cuestionario que recogía información sociodemográfica, datos clínicos y aspectos psicológicos (estado de ánimo, dificultad para afrontar e historia previa de ansiedad o depresión) asociados a las necesidades percibidas tras el diagnóstico, y que administraba el propio oncólogo en los meses inmediatos a la comunicación de la enfermedad.

Resultados: Un $\mathbf{2 4 \%}$ de los pacientes formularon deseo de atención psicooncológica. Se observó que los pacientes más jóvenes, que presentaban alguna alteración emocional, o que no se encontraban preparados para abordar sin mucho esfuerzo la situación, eran los que expresaban mayor interés junto con los pacientes sin antecedentes de diagnóstico y/o tratamien-

\section{Abstract}

Purpose: To state the rate of patients who would ask for psychological assistance, once they have known their diagnosis and treatment assigned. To find the profile of these patients considering their demographic, clinical and psychosocial features.

Method: A sample of 236 patients assisted at Consorci Sanitari de Terrassa (CST) was assessed using a questionnaire which asked for demographic and social features, as well as for some psychological variables (mood states, coping problems, previous anxiety or depression disorders) during the months which followed the diagnosis.

Results: $24 \%$ of patients were interested in receiving psychological assistance. These patients were younger, expressed mood alterations and coping difficulties, and did not have previous anxiety or depression disorders. There were no differences between patients who asked for psychological assistance and those who do not, in gender, educational level, social relationships, diagnostic, or prognosis. Months from the knowledge of diagnosis were also unrelated with desire of receiving psychological assistance.

Correspondencia:

Tomás Blasco Blasco

Departament de Psicologia Bàsica, Evolutiva i de I' Educació

Universitat Autònoma de Barcelona

08193 Bellaterra (Barcelona)

E-mail: tomas.blasco@uab.es 
to psiquiátrico y/o psicofarmacológico. No se encontraron diferencias estadísticamente significativas respecto al género, nivel educativo, pronóstico, el hecho de vivir sólo, tipo de tumor, tiempo transcurrido desde el diagnóstico o grado de diseminación del tumor.

Conclusiones: Se confirma que los pacientes que presentan malestar emocional, pero sin antecedentes de trastorno psicológico, son los que principalmente desean ayuda psicológica así como que las variables clínicas y sociodemográficas no tienen una clara relación con este deseo. Se advierte la necesidad de definir con más precisión qué se entiende por "ayuda psicológica" para que el paciente pueda valorar mejor si lo que se le ofrece puede serle útil para responder a sus necesidades.

Palabras Clave: Predictores, ayuda psicooncológica, perfil demandante.
Conclusions: A definition of what does mean "psychological assistance" is needed in order to understand why only $22 \%$ of patients ask for this resource. Sociodemographic and clinical variables were unrelated with desire of receiving psychological assistance. Younger patients with psychological distress and without previous history of emotional disturbance are more prone to ask for psychological assistance.

Key words: Predictors, psychological assistance, patients demands profile.

\section{INTRODUCCIÓN}

A pesar de que la prevalencia de malestar emocional entre los pacientes oncológicos puede darse en un tercio de ellos (o ser incluso superior en determinadas fases de la enfermedad), sólo una pequeña parte de los mismos desea solicitar atención psicooncológica ${ }^{(1)}$.

Así, en un estudio con 238 pacientes con cáncer de próstata, Voerman et al.,(2) observaron que 136 pacientes no estuvieron interesados en participar en grupos de apoyo, que 55 pacientes estuvieron interesados, pero que no llegaron a participar, y que sólo 47 pacientes interesados llegaron, finalmente, a participar en dichos grupos. Por su parte, Baker-Glenn et al.,(3), en un grupo de 128 pacientes que iban a iniciar quimioterapia observaron que sólo un $20 \%$ de los pacientes que no mostraban malestar emocional deseaban recibir ayuda psicológica, aumentando hasta un 36\% en el caso de los pacientes que cumplían los criterios de malestar emocional, según su puntuación en el HADS. Merckaert et al., ${ }^{(4)}$, en una muestra de 381 pacientes registraron que un $26 \%$ de las mujeres manifestaban deseo de recibir apoyo psicológico, por sólo un $11 \%$ en el caso de los pacientes varones.

Puede sorprender el bajo porcentaje de pacientes que estarían interesados en solicitar apoyo psicológico, pero es difícil establecer una razón que explique este hecho, ya que los resultados de los estudios antes citados se han realizado en poblaciones de pacientes con importantes diferencias entre sí. Se ha evaluado a pacientes con diversos diagnósticos y en diversos momentos del ciclo oncológico ${ }^{(4,5)}$; pacientes en diferentes momentos del ciclo oncológico, aunque con un diagnóstico específico, como próstata ${ }^{(2)}$ o melanoma(6); o pacientes en un momento concreto del ciclo oncológico, como es el diagnóstico, pero con diferentes tipos de tumores ${ }^{(7)}$. Sólo hemos encontrado un trabajo ${ }^{(8)}$ que analiza a pacientes con el mismo tipo de tumor (mama) y en el mismo momento del ciclo oncológico (después de la cirugía).

Desde la perspectiva de una optimización de los servicios asistenciales, sería interesante poder identificar a los pacientes receptivos a una oferta de ayuda psicológica y, en la medida de lo po- 
sible, establecer un perfil o perfiles de sus necesidades y de las estrategias de intervención más adecuadas para satisfacerlas. Hay que descartar, en principio, que el sólo hecho de padecer malestar emocional sea un indicador claro de esa proclividad hacia la recepción de ayuda psicológica. El trabajo anteriormente citado de Baker-Glenn et al., ${ }^{(3)}$ señala que sólo un $36 \%$ de esos pacientes desean recibir ayuda psicológica y, por otro lado, el estudio de Winzer et al., ${ }^{(6)}$ identifica a pacientes que aún experimentando malestar psicológico (en base a criterios de screening) rechazan la recepción de ayuda psicológica (pacientes a los que denominan "avoiders"), mientras que otros (a los que denominan "help-seekers") que no cumplen los criterios de padecer malestar psicológico, sí desearían recibir dicha ayuda. Es posible que deban tenerse en cuenta otros factores a la hora de establecer la tipología de los pacientes que desearían recibir atención psicológica y que, posiblemente, una interacción de factores sociodemográficos, psicológicos y biomédicos pueda ser más orientativa para establecer esa tipología. Así, Winzer et al., ${ }^{(6)}$ señalan que dicho deseo es expresado por los pacientes más jóvenes, por los pacientes que no son evitadores, y por los pacientes que no tienen pareja. Por su parte, Merckaert et al.,(4) identifican que los pacientes que expresan este deseo son los más jóvenes, las mujeres y los que utilizan un estilo de afrontamiento que busca el apoyo. Finalmente, León et al.,(7) señalan que el deseo de recibir ayuda psicológica es más solicitado por las mujeres y por los pacientes que expresaban tener más dificultad para afrontar el cáncer. Complementando estos datos, hay diversos trabajos ${ }^{(2,5,8,9)}$ que indican que los factores más consistentemente relacionados con la participación en la atención psicooncológica son la edad (pacientes más jóvenes), el género (ser mujer), la existen- cia de dificultades para seguir adelante, y el uso de estrategias de afrontamiento basadas en la búsqueda de apoyo.

Como puede verse, los datos no son aún suficientemente concluyentes y es necesario realizar más investigaciones que permitan clarificar tanto el porcentaje de pacientes que desean este tipo de atención, como las características (sociodemográficas, psicológicas, y biomédicas) de los mismos. Un mejor conocimiento de estos pacientes puede permitir un diseño más adecuado de la oferta de atención psicooncológica, ajustando los protocolos asistenciales para que puedan llegar a los enfermos más susceptibles de recibir este tipo de atención en los diferentes momentos del ciclo oncológico. Por esta razón, el objetivo de la presente investigación es conocer la prevalencia de pacientes que, tras ser informados del diagnóstico oncológico y del tratamiento médico prescrito, desearían recibir atención psicooncológica, e identificar las características sociodemográficas, biomédicas y psicológicas de los mismos, con la finalidad de establecer un posible perfil del demandante de atención psicooncológica.

\section{MÉTODO}

\section{Participantes}

Desde septiembre de 2009 hasta enero de 2011 se incluyeron todos los sujetos diagnosticados de cáncer -respetando el impacto y reacción inicial que supone la comunicación de la enfermedad y el tiempo y espacio interior necesario para que el paciente fuese capaz de comprender el tratamiento propuesto- por el servicio de oncología del Consorci Sanitari de Terrassa (CST) y que habían firmado el correspondiente consentimiento informado para participar en el estudio. El CST atiende a una población de referencia de 250.000 habitantes. 


\section{Evaluación y Procedimiento}

Se elaboró un cuestionario que recogía información sociodemográfica, datos clínicos de los sujetos y aspectos psicológicos asociados a las necesidades percibidas. La información psicológica valoraba, mediante una escala Likert de cuatro categorías de respuesta (Mucho, Bastante, Poco, Nada), el grado de malestar emocional que habían tenido durante la última semana (estado de ánimo, nerviosismo, miedo y enfado). Con la misma escala se evaluaba también el grado en que el paciente se sentía preparado para abordar la situación, el esfuerzo que le conllevaba afrontar la situación, y el grado en que creía que podría ayudarle el recibir apoyo psicológico. Después, el paciente respondía SI o NO a si había estado anteriormente en tratamiento con un psiquiatra o psicólogo, si había tenido anteriormente un diagnóstico de ansiedad o depresión, o si había tomado medicación para ese tipo de problemas. Finalmente, el paciente respondía con $\mathrm{SI}, \mathrm{NO}$ o "Me lo pensaré", a la pregunta de si le gustaría recibir ayuda psicológica. La selección de los pacientes candidatos a estudio era realizada por el oncólogo. El cuestionario era auto-administrado, a excepción de la parte clínica que era cumplimentada por el oncólogo, durante la segunda o tercera visita clínica. Esto ocurrió en la mayoría de los casos y dentro de los dos meses posteriores al diagnóstico (199 casos, $84,3 \%$ ). Sin embargo, se podía dar la circunstancia de que algunos de los pacientes fueran diagnosticados por primera vez por otros servicios médicos y derivados al Servicio de Oncología para la valoración del tratamiento a seguir, lo que justificaría los 37 casos en los que el paciente ha respondido al cuestionario cuando habían pasado más de dos meses desde el momento del diagnóstico.

\section{Análisis estadístico}

Se realizó un análisis descriptivo presentando los resultados en frecuencias relativas para las variables cualitativas y la media y rango para las variables cuantitativas. Para la comparación entre variables cualitativas se utilizó la chi-cuadrado y la corrección de Fisher, agrupando los valores de la Escala Likert en dos: "Mucho" y "Bastante" versus "Poco" y "Nada". Para la comparación entre variables cuantitativas se utilizó el análisis de la varianza del programa estadístico SPSS versión 19.0 y el nivel de significación estadístico considerado del $5 \%$.

\section{RESULTADOS}

Cumplieron criterios de inclusión y dieron su consentimiento para participar en el estudio 236 pacientes. La media de edad fue de 61 años $\left(\mathrm{IC}_{95}: 59-62\right)$, un $51,3 \%$ (121) eran mujeres, y un $85,6 \%$ (202) vivían acompañados. El 34\% (83) tenía estudios medios o universitarios. Respecto a las variables clínicas, están resumidas en la tabla 1 . El tumor más frecuente es el de mama y el grado de diseminación más común es el locorregional.

Las características emocionales de los pacientes están indicadas en la tabla 2 y aunque más del $50 \%$ de los pacientes señala estar preparado para abordar la situación invirtiendo poco esfuerzo, se observa que un $68,6 \%$ (162) presentan desánimo, un $53,4 \%$ (126) nerviosismo, un $74,5 \%$ (176) están enfadados y que cerca del $80 \%$ tienen miedo. En relación a los antecedentes emocionales un $16,1 \%$ (38) había estado en tratamiento con un psiquiatra o psicólogo con anterioridad, un 25,4\% (60) tenían un diagnóstico previo de depresión y/o ansiedad y un $27,1 \%$ (64) habían tomado medicación para la depresión. 
Tabla 1. Características clínicas de la muestra.

\begin{tabular}{|r|c|c|}
\cline { 2 - 3 } \multicolumn{1}{c|}{} & \multicolumn{2}{c|}{ Resultados } \\
\hline TRATAMIENTO PREVISTO & $\mathbf{\%}$ & $\mathbf{N}$ \\
\hline Quimioterapia & 48,3 & 114 \\
\hline Radioterapia & 2,5 & 6 \\
\hline Otros & 16,5 & 39 \\
\hline Ninguno & 4,7 & 11 \\
\hline Quimioterapia y Radioterapia & 26,3 & 62 \\
\hline PRONÓSTICO A PRIORI & $\mathbf{\%}$ & $\mathbf{N}$ \\
\hline 1-3 años & 21,6 & 51 \\
\hline$>3$ años & 25,8 & 61 \\
\hline CoCALIZACIÓN TUMOR & $\mathbf{\%}$ & $\mathbf{N}$ \\
\hline Mama & 27,1 & 64 \\
\hline Colorrectal & 24,2 & 57 \\
\hline Pulmón & 20,3 & 48 \\
\hline Otros GI & 10,2 & 24 \\
\hline
\end{tabular}

\begin{tabular}{|r|c|c|}
\hline GRADO DISEMINACIÓN TUMOR & $\mathbf{\%}$ & $\mathbf{N}$ \\
\hline Local & 24,6 & 58 \\
\hline Locorregional & 33,9 & 80 \\
\hline Metastásico & 26,7 & 63 \\
\hline
\end{tabular}

\begin{tabular}{|r|c|c|}
\hline TIEMPO DESDE DIAGNÓSTICO & $\mathbf{\%}$ & $\mathbf{N}$ \\
\hline$<\mathbf{1}$ mes & 19,5 & 46 \\
\hline $\mathbf{1 - 2}$ meses & 64,8 & 153 \\
\hline $\mathbf{3 - 1 1}$ meses & 11,4 & 27 \\
\hline$>\mathbf{1 1}$ meses & 3 & 7 \\
\hline
\end{tabular}

Tabla 2. Estado emocional de los pacientes.

\begin{tabular}{|r|c|c|c|c|}
\cline { 2 - 5 } \multicolumn{1}{c|}{} & \multicolumn{2}{c|}{ Mucho/Bastante } & \multicolumn{2}{c|}{ Poco/Nada } \\
\hline ESTADO EMOCIONAL & $\mathbf{\%}$ & $\mathbf{N}$ & $\mathbf{\%}$ & $\mathbf{N}$ \\
\hline Desanimado & 68,6 & 162 & 31,4 & 74 \\
\hline Nervioso & 53,4 & 126 & 46,6 & 110 \\
\hline Miedo & 77,5 & 183 & 22,5 & 53 \\
\hline Enfado & 74,5 & 176 & 25,5 & 60 \\
\hline Grado de esfuerzo para abordar la situación & 62,7 & 148 & 37,3 & 88 \\
\hline
\end{tabular}


Finalmente, un 39\% (92) manifiesta que no cree que la ayuda psicológica mejore su situación, y respecto al deseo de recibirla, un $41,5 \%$ (98) indica que no le gustaría, un 33,9\% (80) responde que se lo pensará y un 24,2\% (57) afirma que sí le gustaría poder acceder a ella.

Respecto a los factores relacionados con el deseo de recibir ayuda psicológica, se observa que los pacientes que lo manifiestan son más jóvenes $(M=56,63)$ que los que no la desean $(M=63,36)$, siendo la diferencia estadísticamente significativa $(p=0,002)$. Asimismo, un 58\% de los pacientes que manifiestan desánimo desean recibir ayuda psicológica, por sólo un 26\% de los que no muestran desánimo, siendo esta diferencia estadísticamente significativa $(p<0,001)$. Lo mismo sucede respecto al nivel de nerviosismo $(p=0,007)$ donde los pacientes con altos niveles desean ayuda psicológica en un 47\% de casos, por sólo un $27 \%$ en los pacientes con niveles bajos. También hay diferencias significativas respecto al miedo $(p=0,001)$, pues un $62 \%$ de pacientes con niveles altos de miedo desean ayuda psicológica por sólo un 30\% de los que dicen tener poco miedo. Por último, las diferencias también se dan en función del nivel de enfado $(p<0,001)$, deseando ayuda psicológica un $65 \%$ de pacientes que tienen niveles altos de enfado por sólo un $27 \%$ de los pacientes cuyo nivel de enfado es bajo.

Estos resultados se repiten cuando consideramos el nivel de preparación para afrontar la situación que tienen los pacientes $(p=0,01)$, ya que el $56 \%$ de los pacientes que no se encuentran preparados desea asistencia psicológica, por sólo un 31\% de los pacientes que sí dicen estar preparados. Lo mismo puede decirse del esfuerzo por afrontar la situación $(p<0,001)$, ya que los pacientes que invierten más esfuerzo desean más la ayuda psicológica (un 67\% de los casos) que los que invierten un esfuerzo menor (21\% de los casos).
En relación al tratamiento psiquiátrico o psicológico previo a la enfermedad, un $70,2 \%$ de los pacientes que no han recibido tratamiento desean asistencia psicológica frente a un $29,8 \%$ de los pacientes que si que han estado en tratamiento psiquiátrico, siendo las diferencias estadísticamente significativas $(p=0,002)$.

Un $64,9 \%$ de los pacientes sin diagnóstico previo de depresión o ansiedad desean más ayuda psicológica que los que ya han sido diagnosticados de algún trastorno emocional $(35,1 \%, p=0,005)$. La misma relación se observa en los pacientes que tomaban medicación para su estado emocional, un 42,1\% desean ayuda psicológica respecto a un $57,9 \%$ de los que no están en tratamiento $(p=0,001)$.

No se han encontrado diferencias estadísticamente significativas entre los que desean recibir ayuda y los que no respecto al género, nivel educativo, pronóstico, el hecho de vivir sólo, tipo de tumor, tiempo transcurrido desde el diagnóstico, o grado de diseminación del tumor.

\section{DISCUSIÓN}

En el presente trabajo se ha valorado la prevalencia de pacientes que desean recibir ayuda psicológica -una vez diagnosticados y conociendo el tratamiento que deberán seguir a lo largo del proceso de la enfermedad- y se han analizado diferentes factores que podrían estar relacionados con el deseo de solicitar ayuda psicológica. Los resultados obtenidos indican que un $24 \%$ de los pacientes formula esta demanda y que su perfil indica que son más jóvenes, que se ven poco preparados para afrontar la situación, que han de invertir mucho esfuerzo en ese afrontamiento, y que muestran alteración emocional.

Asimismo, los datos indican que las personas que no han estado previamente diagnosticadas de alteración emocional, no han recibido ningún tipo de tratamien- 
to psicológico o psiquiátrico y no han tomado psicofármacos, presentan un mayor deseo de soporte psicoterapéutico frente al diagnóstico de la enfermedad. Sin embargo, el deseo de recibir ayuda psicológica no guarda relación con el hecho de ser hombre o mujer, el nivel educativo, o vivir solo, ni tampoco con las variables clínicas relativas al tipo de tumor y su grado de diseminación, pronóstico, o tiempo transcurrido desde el diagnóstico.

El porcentaje de pacientes que desean ayuda psicológica es similar al encontrado en otros trabajos y que oscilan entre el $20 \%-30 \%{ }^{(2,3)}$. Una razón para entender estas cifras es que, posiblemente, los pacientes pueden tener diferentes expectativas respecto a lo que es una intervención psicológica y cómo se lleva a cabo. Nuestros datos indican que hay una mayoría de pacientes (58\%) que cree que el apoyo psicológico podría ayudarle, pero que sólo desean recibirlo un $24 \%$, mientras que un $34 \%$ dice que se lo pensará. Debe tenerse en cuenta que no existe una definición clara ni un consenso completo sobre lo que se entiende por "intervención psicológi$\mathrm{ca}^{\prime \prime(10)} \mathrm{y}$, por otra parte, es evidente que no es lo mismo una atención individualizada, dentro de un protocolo de interconsulta en una institución hospitalaria, que una participación en grupos de apoyo fuera del entorno del hospital en el que es tratado el paciente, ya que pacientes que demandarían una asistencia del primer tipo, pueden ser reacios a participar en una del segundo tipo y viceversa. Lo que sí parece claro es que hay alrededor de un $42 \%$ de pacientes que no desean recibir ayuda psicológica y que no creen que pueda serles de utilidad.

De los factores relacionados con el deseo de recibir ayuda psicológica encontrados en el presente trabajo, la edad ha sido el que también ha aparecido con más frecuencia en la literatura ${ }^{(4-6)}$ siendo los pacientes más jóvenes los que expresan ese deseo. Esto puede deberse a que este tipo de población conoce mejor lo que puede aportar la asistencia psicológica, y que personas con más edad lo desconocen o lo asocian directamente con la idea de padecer enfermedad mental, lo que puede ocasionar un claro rechazo. Aun cuando podría pensarse que esto debería ir relacionado con el nivel de estudios, nuestros datos no confirman esta hipótesis, a pesar de que en un trabajo previo(5) sí se encontró esta relación. Tampoco hemos encontrado relación entre el hecho de vivir solo y el deseo de recibir ayuda psicológica, en línea con el trabajo de Voerman et al., ${ }^{(2)}$ aún cuando otros trabajos no corroboran este hallazgo ${ }^{(5)}$. De todas formas, esta variable puede no ser adecuada si con ello se pretende valorar la existencia de apoyo social, ya que el hecho de vivir solo no implica necesariamente que no exista una red de relaciones sociales que ayude a la persona a sobrellevar su enfermedad.

Nuestros resultados indican que no hay relación entre el deseo de recibir ayuda psicológica y el ser hombre o mujer. Ello se inscribe en el marco de una literatura que muestra resultados contradictorios. Merckaert et al., ${ }^{(4)}$ y Grande et al., ${ }^{(5)}$ señalan que la demanda de ayuda psicológica se da más en mujeres, mientras que Winzer et al., ${ }^{(6)}$, en un grupo de pacientes con melanoma, encuentra que la ayuda psicológica es más solicitada por los hombres. El papel de la variable sexo no queda, pues, suficientemente claro. Es posible que realmente hombres y mujeres tengan la misma prevalencia de deseo de recibir ayuda psicológica, como sugieren nuestros resultados, y que algunos trabajos no hayan replicado este dato porque los hombres no manifiestan este deseo porque quizá perciban que la oferta de dicha ayuda no responda a sus demandas específicas.

Respecto a las variables clínicas, nuestros resultados indican que no hay diferencias entre los tres principales tipos de tumores (mama, colon y pulmón) respecto al 
deseo de recibir ayuda psicológica, ni tampoco respecto al grado de diseminación del mismo. En la literatura previa no hemos encontrado trabajos que establecieran diferencias entre los distintos tipos de diagnóstico, cuando se han evaluado muestras de pacientes con diferentes tumores. El grado de diseminación del tumor tampoco se ha relacionado con el deseo de ayuda psicológica en pacientes de próstata ${ }^{(2)} \mathrm{O}$ de melanoma $^{(6)}$. El tipo de tratamiento previsto tampoco se ha relacionado con el deseo de ayuda psicológica ${ }^{(2)}$. Sin embargo, en el caso de los pacientes que son derivados por otros profesionales predominan los que tienen la enfermedad más avanzada o un peor pronóstico ${ }^{(11,12)}$. Parece, pues, que las variables clínicas cobran relevancia cuando la demanda de ayuda la formulan otros profesionales, pero que no son adecuadas para identificar a los pacientes que la realizan por su cuenta.

Respecto al tiempo transcurrido desde el diagnóstico, tampoco hemos encontrado que se relacione con el deseo de recibir ayuda psicológica. Al respecto, la literatura proporciona datos poco congruentes. Así, Voerman et al., ${ }^{(2)}$ no encuentran diferencias en esa variable entre los que participaron en un programa de ayuda psicológica y los que no lo hicieron. En cambio, el estudio de Nekolaichuk et al.,(1) sí encuentra, en pacientes referidos, que el tiempo desde el diagnóstico era más breve. En un estudio realizado en nuestro país ${ }^{(13)}$ se observó que los pacientes y sus familiares tardaban entre 8 y 9 meses desde el momento del diagnóstico en solicitar ayuda psicooncológica, y que los que tardaban menos tiempo eran aquellos que tenían más apoyo social, tardando más los pacientes que consideraban que no necesitaban la ayuda, aunque en bastantes casos ocurría que la demora en solicitar asistencia estaba ocasionada por desconocer la posibilidad de acceder a este servicio. Parece, pues, que el tiempo transcurrido desde el diagnóstico no es una variable que permita identificar claramente a los pacientes que desean recibir ayuda psicológica.

Nuestros resultados indican que las cuatro variables a través de las que se ha pretendido valorar el malestar psicológico (tristeza, nerviosismo, miedo y enfado) coinciden en señalar que los pacientes que expresan deseo de recibir ayuda psicológica muestran mayor incidencia de dichos estados de ánimo. La literatura no es totalmente clara en este sentido, ya que hay trabajos que no encuentran relación entre el nivel de distrés y el deseo de solicitar ayuda psicológica en pacientes con cáncer de mama ${ }^{(8)} \mathrm{o}$ de melanoma ${ }^{(6)}$, mientras que en investigaciones con pacientes de diferentes tumores hay estudios que sí encuentran esa relación ${ }^{(13)}$, pero otros $\mathrm{no}^{(4)}$. Estas diferencias podrían deberse a que los investigadores utilizan diferentes instrumentos y criterios a la hora de evaluar el nivel de distrés, ya que, por ejemplo, algunos utilizan el HAD y otros utilizan el termómetro del distrés. Es posible que el nivel percibido de esfuerzo por seguir adelante pueda ser un indicador más claro, tal como hemos observado en un trabajo previo $^{(7)}$, y como también parece sugerir el trabajo de Merckaert et al., ${ }^{(4)}$.

El deseo de recibir ayuda psicológica es manifestado en mayor medida en pacientes que no tienen antecedentes de tratamiento psiquiátrico, diagnóstico de depresión o ingesta de medicación por dichos motivos. Esto puede parecer paradójico ya que cabe pensar que la existencia de malestar emocional previo al diagnóstico de cáncer facilitaría la demanda de asistencia psicológica. No obstante, podría explicarse porque quizá estas personas disponen ya de un referente profesional que puede ofrecerles la ayuda que requieran y por ello rechazan la que se les oferta. En cambio, algunos de los pacientes que nunca han estado diagnosticados de alteración emocional, ni se han medicado, ni trata- 
do al efecto, podrían, ante una situación en la que se sienten especialmente vulnerables, expresar su deseo de tener este tipo de soporte con la finalidad de sentirse protegidos, lo que podría argumentar que los porcentajes resultantes de necesidad de ayuda sean más altos en este grupo de pacientes. En cualquier caso, es una variable cuyo potencial como predictor debe investigarse más a fondo, pues no hemos encontrado que haya sido evaluada en ninguna de las investigaciones que hemos podido consultar.

Finalmente, hay que tener en cuenta que una variable no contemplada en el presente estudio como es el tipo de afrontamiento empleado por los pacientes también puede estar relacionada con el deseo de ayuda psicológica. Merckaert et al., ${ }^{(4)}$ señalan que desean ayuda aquellas personas que tienen un estilo de afrontamiento de búsqueda de apoyo y Grande et al., ${ }^{(5)}$ señalan que los pacientes que participan tienen más apoyo social y usan más estrategias de afrontamiento activas. Por su parte, Schou et al., ${ }^{(8)}$ dicen que la única variable que diferencia a las pacientes con cáncer de mama que acuden a un programa de ayuda psicológica es el optimismo (las que asistieron a la intervención eran más optimistas), por lo que es posible que esta variable también pueda ser adecuada para identificar pacientes que desean recibir atención psicológica.

A pesar de que el número de pacientes evaluado en la presente investigación es considerable, quizá no es suficiente para poder evaluar de forma simultánea la intensidad de la relación de cada una de las variables que pueden estar ligadas al deseo de recibir ayuda psicológica, así que convendría realizar estudios con muestras más amplias o bien restringir el tipo de tumor a evaluar de cara a obtener grupos más homogéneos de pacientes que, presuntamente, pueden mostrar perfiles de necesidades asistenciales más similares.
Por otra parte, debería también incluirse, en la medida de lo posible, una evaluación de los recursos de afrontamiento que el/la paciente posee, a fin de poder evaluar si dichos recursos son también una variable que juega un papel a la hora de establecer la necesidad de una atención psicológica.

\section{IMPLICACIONES CLÍNICAS}

Creemos que es necesario definir con más precisión qué se entiende por "ayuda psicológica" para que el paciente pueda valorar mejor si lo que se le ofrece puede serle útil para responder a sus necesidades. Esto podría ser útil no sólo para los pacientes indecisos, sino también para que aquellos que rechazan la ayuda psicológica porque no creen en ella puedan asegurarse de que efectivamente ese apoyo no es pertinente a su caso. Probablemente, la estrategia adecuada a seguir es la de detectar las necesidades específicas de cada paciente o grupo de pacientes que comparten la misma situación oncológica y valorar en qué grado realmente se desea una intervención psicológica claramente definida que realmente sea susceptible de satisfacer dichas necesidades.

Nuestro estudio ha confirmado algunos trabajos anteriores en los que se había visto que las variables clínicas no tienen una relación clara con el deseo de recibir ayuda psicológica expresado por el paciente. Asimismo, también confirma que el malestar emocional es más frecuente en los pacientes que desean ayuda, pero que el hecho de padecer dicho malestar no necesariamente determina la demanda de asistencia psicológica, por lo que es preciso utilizar, además de ésta, otras variables psicológicas del/la paciente.

\section{REFERENCIAS BIBLIOGRÁFICAS}

1. Nekolaichuk C, Cumming C, Turner J, Yushchyshyn A, Sela R. Referral patterns 
and psychosocial distress in cancer patients accessing a psycho-oncology counseling service. Psychooncology 2011;20:32632. Doi:10.1002/pon. 1765

2. Voerman B, Visser A, Fischer M, Garssen B, Van Andel G, Bensing J. Determinants of participation in social support groups for prostate cancer patients. Psychooncology 2007; 16:1092-7. Doi:10.1002/ pon. 1160

3. Baker-Glenn E, Park B, Granger L, Symonds P, Mitchell A. Desire for psychological support in cancer patients with depression or distress: Validation of a simple help question. Psychooncology 2011; 20: 525-31. Doi:10.1002/pon.1759

4. Merckaert I, Libert Y, Messin S, Milani M, Slachmuylder JL, Razavi D. Cancer patients' desire for psychological support: Prevalence and implications for screening patients'psychological needs. Psychooncology 2010; 19:141-9. Doi:10.1002/ pon. 1568

5. Grande GE, Myers LB, Sutton SR. How do patients who participate in cancer support groups differ from those who do not? Psychooncology 2006;15:321-34. Doi:10.1002/pon.956

6. Winzer A, Hoppe A, Altenhoff J, Kuwert $C$, Koch $U$, Schulz $H$. Interest in a psycho educational group intervention among out-patients with malignant melanoma in relation to their need: Which patients are likely to participate? Psychooncology 2009;18:1179-88. Doi:10.1002/pon.1164

7. Leon C, Jovell E, Arenas G, Alfaro J, Blanco $\mathrm{R}$, Pisa A, et al. Gender differences in dealing with emotional aspects and the desire to receive professional psychological help, in cancer patients at the first visit to an Oncology Service. Póster. IPOS $12^{\text {th }}$ World Congress of Psycho-Oncology, Quebec City,Canada, 25-29 mayo 2010.

8. Schou I, Ekeberg O, Karesen R, Sorensen E. Psychosocial intervention as component of routine breast cancer care 3 who participates and does it help? Psychooncology 2008; 17: 716-20. Doi:10.1002/pon.1264

9. Sanchez N, Torres A, Camprubí N, Vidal A, Salamero M. Factores predictores del abandono de la intervención psicológica grupal en una muestra de pacientes con cáncer de mama. Psicooncología, 2005;2(1):21-32.

10. Hodges L, Walker J, Kleiboer AM, Ramirez AJ, Richardson A, Velikova G, et al. What is a psychological intervention? A metareview and practical proposal. Psychooncology 2011;20:470-8. Doi:10.1002/ pon. 1780

11. Almonacid V, Moreno P, Lluch, A. Estudio sobre la derivación de pacientes oncológicos a la unidad de Psicooncología según criterio médico. Psicooncología 2009; 6(1):203-10.

12. Hernández M, Cruzado JA, Arana, Z. Problemas psicológicos en pacientes con cáncer: dificultades de detección y derivación al psicooncólogo. Psicooncología 2007; 4(1):179-91.

13. Aresté $\mathrm{V}$, García-Lumbreras S. Tiempo y motivos de demora en la demanda de atención psicológica en pacientes oncológicos y familiares. Póster. 10th World Congress of the IPOS. Madrid, 9-13 junio 2011. 